\title{
Clinical, morphological and genetic features of a cohort of late onset GSD II patients: typical and atypical presentations
}

\author{
E Barca*, O Musumeci, C Rodolico, A Ciranni, G Vita, A Toscano \\ From Proceedings of the 6th European Symposium: Steps Forward in Pompe Disease \\ Berlin, Germany. 23-24 November 2012
}

\section{Introduction}

GSDII (Pompe disease) is a rare, autosomal recessive disease due to alpha-glucosidase (GAA) deficiency that presents with infantile and late-onset forms. Herein, we describe a cohort of 29 late-onset patients (15 males and 14 females, aged 9 to 76 ), and discuss some unusual clinical features seen in 4 of them.

\section{Methods}

Clinical evaluations were performed in all patients using the Walton and Gardner-Medwin, MRC and GSGC scales, and the 6MWT. Respiratory function was assessed using $\mathrm{FVC} \%$ in upright and supine positions. All patients underwent morphological and biochemical examinations, muscle MRI, neurophysiological studies, and molecular genetic analysis.

\section{Results}

Out of 29 patients, 15 received enzyme replacement therapy (ERT). 57\% initially had limb-girdle involvement, $29 \%$ an isolated hyperCKemia, and $14 \%$ respiratory insufficiency. Neurophysiological studies revealed a myopathic pattern in $48 \%$ of patients, neurogenic or mixed in $28 \%$, whereas $24 \%$ of patients did not show any electrical abnormality. In the examined patients, muscle MRI showed early involvement of ileopsoas, gluteus and posterior thigh muscles. Morphological studies revealed vacuolar myopathy in $68 \%$ of patients, and biochemical analysis showed residual alpha-glucosidase activity ranging from $0.01 \%$ to $25 \%$. Molecular genetic analysis confirmed IVS13T $>\mathrm{G}$ as the most common mutation, but we also identified three novel point mutations. Atypical clinical features were observed in 4 out of 29 patients: a 66-year-old female with disease onset involving distal upper limbs muscles; a 70-year-old male with mesial temporal sclerosis without epileptic or cognitive disorders; a 46-year-old male with severe neurosensory hearing loss (with very early hearing difficulties); and a 51-year-old female with a congenital absence of a thyroid lobe.

\section{Conclusion}

Our study confirms the clinical, morphological and molecular genetic heterogeneity of late-onset GSDII. Muscle MRI is a very useful tool for muscle damage evaluation, especially at the early stages of the disease. Based on our experience with some atypical cases, we suggest always performing a complete clinical and laboratory evaluation of patients in order to highlight unusual muscular (e.g. distal involvement) and/or CNS presentations. These cases reinforce the hypothesis that modifier genes and/or epigenetic factors may contribute to clinical presentations of Pompe disease.

Published: 29 May 2013

doi:10.1186/1471-2474-14-S2-P9

Cite this article as: Barca et al: Clinical, morphological and genetic features of a cohort of late onset GSD II patients: typical and atypical presentations. BMC Musculoskeletal Disorders 2013 14(Suppl 2):P9.

Department of Neurosciences, University of Messina, Messina, Italy

( 2013 Barca et al; licensee BioMed Central Ltd. This is an Open Access article distributed under the terms of the Creative Commons 